\title{
The influence of deep traps on transient current-voltage characteristics of organic light-emitting diodes
}

\author{
P.H. Nguyen ${ }^{a *}$, S. Scheinert ${ }^{b}$, S. Berleb ${ }^{a}$, W. Brütting ${ }^{a}$ and G. Paasch ${ }^{c \dagger}$ \\ ${ }^{a}$ University of Bayreuth, Experimental Physics II, D-95440 Bayreuth, \\ Germany \\ ${ }^{b}$ Technical University of Ilmenau, Solid State Electronics, D-98684 \\ Ilmenau, Germany \\ ${ }^{c}$ Institute for Solid State and Materials Research Dresden, D-01171 \\ Dresden, Germany
}

\begin{abstract}
Transient current-voltage characteristics of organic light-emitting diodes (OLEDs) made from both conjugated polymers and low molecular-weight materials show hysteresis effects in the reverse bias regime depending on the direction and speed of the bias sweep. This behaviour is quantitatively investigated here for the example of devices based on N,N'-diphenyl-N,N'-bis(1naphtyl)-1,1'-biphenyl-4,4'-diamine (NPB) with Ca and indium-tin oxide as electrodes. To clarify the origin of this peculiarity numerical simulations have been carried out supposing the existence of deep acceptor-like trap states. Typical trends are shown by systematically varying parameters such as measuring conditions, trap characteristics, basic doping level, mobility and injec-
\end{abstract}

\footnotetext{
*Present address: Institute of Semiconductor Technology, University of Hannover, Appelstraße 11A, D-30167 Hannover, Germany. E-Mail: nguyen@iht.uni-hannover.de

${ }^{\dagger}$ Corresponding author, E-Mail: g.paasch@ifw-dresden.de
} 
tion conditions. Based on the simulated potential and concentration profiles it is shown that the hysteresis of the current-voltage characteristics is caused by recharging of deep traps for holes. It occurs only if the reverse steadystate current is lower than the trap recharging current and if both currents have different bias dependencies. The origin for the large time needed for the traps to relax into the equilibrium state is clarified. In accordance with the high barrier for the holes at the cathode the calculated reverse current is much smaller than the measured one. Using a new analytical expression for the Schottky diode current-voltage characteristics for a low-doped thin film device, it is shown qualitatively that in real devices a leakage current should dominate for reverse bias.

PACS: 72.80.L, 73.61.Ph, 78.60.Fi, 42.70.Jk

\section{INTRODUCTION}

Only about one decade after the pioneering reports on thin film organic light-emitting diodes (OLEDs) [1,2] a fast development led to first commercial display applications based on these devices [3]. Thereby a detailed understanding of the device physics has been

obtained both for OLEDs based on polymers [4] and for those based on low molecularweight organic materials as well as for the now dominating highly efficient multi-layer devices [5]. Nevertheless, there remain a lot of open questions or controversial opinions. E.g. in some papers the current-voltage characteristics is connected with trap-dominated spacecharge limited currents [6-10] whereas it is assumed in other publications that the field dependence of the mobility is dominating [11-15]. Also, different models and assumptions on the energetic distribution of both the transport and trap states have been presented and discussed [6,14,16-19]. The existence of traps has been proved with different experimental techniques and the reported trap energies vary from about $0.1 \mathrm{eV}$ to about $1 \mathrm{eV}$ [20-23].

Until now most of the work has been concentrated on forward bias operation, since 
light emission is the process one is interested in. However, a complete understanding of the electronic processes in the device and of the material properties is achieved only if also for reverse bias the current-voltage characteristics and the ac behavior can be described adequately. Thus, it has been shown recently [24] that detailed information on internal field and charge distributions in hetero-layer devices can be obtained from capacitance-voltage measurements in the reverse bias region. Here we deal with a peculiarity of the transient current-voltage characteristics, which has been observed in OLEDs with different materials, both in single- and multi-layer devices [25-27]: The current-voltage characteristics shows a hysteretic behaviour for reverse and small forward bias (below the steep increase of the current at the built-in voltage). Even for large delay times (of the order of seconds) after each voltage ramp, the current does not vanish at zero bias but at a negative value for a sweep from negative to positive bias and at a positive bias for the reverse sweep direction. It requires delay times up to 100s to approach the steady-state current. It has been supposed in some publications [25-27] that deep traps might be responsible for this behaviour. If this were the case, one should further ask what kind of traps and at which energy could cause this effect and how large their concentration should be as compared to a possible unintentional doping. Furthermore, the question should be clarified whether such traps can influence the overall device operation. We note that their influence on capacitance-voltage measurements has also been simulated by us [30]. In addition, in some cases the existence of mobile ions has been discussed $[28,29]$. Thus it is of interest to investigate whether the effect can be explained exclusively by deep traps.

In this paper, extending our recently published results [31], we try to answer these questions. For this purpose the effect has been studied and characterized experimentally in single-layer devices based on the hole conducting material NPB. A principal understanding of the mechanism and the physical origin of this effect is obtained by detailed two-dimensional simulations of the operation of devices with material parameters typical for OLEDs and supposing the existence of deep acceptor-like trap states. 


\section{EXPERIMENTAL}

\section{A. Device fabrication and measurement procedure}

Devices were prepared in a sandwich-like structure on glass substrates precoated with

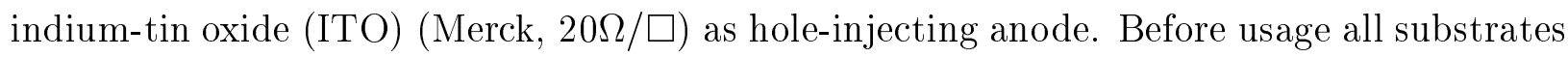
were cleaned by an ultrasonic treatment in deionized water and acetone followed by an oxygen-plasma process which served to remove residual organic contaminants and to enhance the work function of ITO. Immediately after the plasma treatment the substrates were loaded into a nitrogen filled glove box system, from where they could be transferred under inert atmosphere to a high vacuum evaporation system (Leybold). N,N'-diphenyl-N,N'-bis(1naphtyl)-1,1-biphenyl-4,4-diamin (NPB or $\alpha$-NPD) was purchased from Syntec. NPB was deposited on the ITO substrates by thermal evaporation from resistively heated Ta or Mo boats at a base pressure of $10^{-6}$ mbar with a deposition rate of $1 \AA / \mathrm{s}$. The thickness of the organic layer was monitored during deposition by a quartz crystal (Inficon). On top of the NPB layer a 50nm thick Ca layer was deposited in the same chamber without breaking the vacuum. Thereafter the devices were transferred back to the glove box where they were stored and measured under inert conditions $\left(<1 \mathrm{ppm} \mathrm{H}_{2} \mathrm{O},<1 \mathrm{ppm} \mathrm{O}_{2}\right)$. The electrodes were patterned to give four identical devices on one substrate with an area of $4 \times 2 \mathrm{~mm}^{2}$ each.

Current-voltage (I-V) characteristics were measured with a Hewlett Packard semiconductor parameter analyzer (HP 4155B) by means of the integrated stair-case sweep function. To avoid influence of external noise sources measurements were performed in a modified HP $16442 \mathrm{~A}$ test fixture. With this setup currents as low as $10^{-13} \mathrm{~A}$ can be reliably measured. I-V characteristics where measured for both sweep directions (increasing and decreasing voltage) by the procedure displayed in Fig. 1: After application of the starting value the voltage was kept constant for a specified hold time ranging up to 655s. Then the stair-case type voltage sweep was started with each single step consisting of a voltage increment (resp. decrement) by a value $\Delta U$, a delay time $t_{d}$ (ranging from 0 to $65 \mathrm{~s}$ ) during which the voltage 
was kept constant before the current measurement was taken by integrating over a period of 16 power line cycles (equivalent to $320 \mathrm{~ms}$ ). This was repeated until the final value of the voltage sweep was reached. Larger delay times up to 300s were realized with a Keithley 236 source-measure unit following the same procedure. Since the sensitivity of the Keithley 236 was less compared to the HP 4155B these measurements showed a higher noise level.

\section{B. Transient current-voltage characteristics}

As an example we show in Fig. 2 the I-V characteristics of an ITO/NPB/Ca single-layer device with a thickness of $430 \mathrm{~nm}$ measured in steps of $0.1 \mathrm{~V}$. The measurements started at a reverse bias of $U=-7 \mathrm{~V}$ with a stepwise increase to a forward bias of $U=7 \mathrm{~V}$ ('up' sweep), followed by the 'down' sweep in the reverse direction. Here, using a standard measuring procedure the hold time was just the same as the delay time. For a delay time of 0.5s one notices that there is a large hysteresis between both sweep directions below the turnon voltage at about $2 \mathrm{~V}$. The voltage where the current passes through zero is shifted to a negative value for the up sweep and a positive value for the down sweep, respectively. Increasing the delay time to 65 s reduces the hysteresis and also the value of the reverse current, however, to achieve almost identical characteristics in both sweep directions an extremely long delay time of 300 s is required for our devices. Below the turn-on voltage the current is roughly proportional to the applied bias as demonstrated in the inset of the Fig. 2. Locking at the forward bias behaviour it is seen that the steep increase of the current does not begin at $0 \mathrm{~V}$ but at a turn-on voltage of about $2 \mathrm{~V}$. The current increases almost exponentially over many orders of magnitude in a narrow voltage range between 2 and $3 \mathrm{~V}$ and then grows more slowly with the applied bias. The rectification ratio between forward and reserve bias is more than 7 orders of magnitude at $|U|=6 \mathrm{~V}$. In the following we are interested in the hysteresis below the turn-on voltage and hence the measurements are limited to the range between -7 and $3 \mathrm{~V}$ varying the delay time and the bias step. In order to approach equilibrium conditions at the starting point we have now additionally chosen a 
large hold time of $655 \mathrm{~s}$. Fig. 3 shows the I-V characteristics of the same device as in Fig. 2 under these conditions. The bias step $\Delta U=0.1 \mathrm{~V}$ was the same for all curves and $t_{d}$ varied from $0.5 \mathrm{~s}$ to $65 \mathrm{~s}$. The I-V characteristics for the up sweep are depicted in Fig. 3a and the subsequent down sweeps in Fig. 3b. All the I-V characteristics show the same peculiarities as discussed above: below the turn-on voltage at about $2 \mathrm{~V}$ there is a hysteresis and the current grows almost linearly with the applied bias. It is clearly seen in Fig. 3a that for the up sweep the current zero is shifted to higher negative bias with decreasing delay time. With $t_{d}=0.5 \mathrm{~s}$ the current passes through zero already at $U=-6.8 \mathrm{~V}$, with $t_{d}=10 \mathrm{~s}$ at $U=-5.8 \mathrm{~V}$. With a very large delay time of $65 \mathrm{~s}$ the current zero occurs still at $U=-0.4 \mathrm{~V}$. In the down sweep the current zero is shifted to higher positive applied bias with decreasing delay time. With $t_{d}=0.5 \mathrm{~s}$ the current passes through zero at $U=2 \mathrm{~V}$, with $t_{d}=10 \mathrm{~s}$ at $U=1.8 \mathrm{~V}$ and for a very large delay time of $65 \mathrm{~s}$ at $U=0.4 \mathrm{~V}$. Above the turn-on voltage the processes causing the shift of the current zero become negligible and the current is virtually not affected by the different measurement speed. If one assumes that recharging of deep traps is responsible for these observations, one has to expect that the time constant of such processes should be in the order of tens of seconds since the position of the current zero is changed rapidly between delay times of $10 \mathrm{~s}$ and $65 \mathrm{~s}$.

\section{SIMULATION METHOD}

\section{A. Transient current simulation}

Numerical simulations have been carried out using the drift-diffusion model in the twodimensional device simulation program ATLAS [32]. In order to prove the applicability to organic materials, some details need to be discussed. The program solves simultaneously the Poisson equation for the electrical potential $\varphi$ (position dependent intrinsic potential) and the continuity equations for the hole and electron densities, which in the non-degenerate limit are connected with the hole and electron quasi-Fermi potentials $\varphi_{\mathrm{Fp}}$ and $\varphi_{\mathrm{Fn}}$ by 


$$
\begin{aligned}
p & =n_{\mathrm{i}} \exp \left\{\left(\varphi_{\mathrm{Fp}}-\varphi\right) / U_{\mathrm{T}}\right\} \quad, \quad n=n_{\mathrm{i}} \exp \left\{\left(\varphi-\varphi_{\mathrm{Fn}}\right) / U_{\mathrm{T}}\right\} \\
n_{\mathrm{i}} & =\sqrt{N_{\mathrm{V}} N_{\mathrm{C}}} \exp \left\{-\varepsilon_{\mathrm{g}} / 2 \mathrm{e} U_{\mathrm{T}}\right\}
\end{aligned}
$$

The intrinsic density $n_{\mathrm{i}}$ is connected with the gap energy $\varepsilon_{\mathrm{g}}$ and the effective densities of states $N_{\mathrm{V}}$ and $N_{\mathrm{C}} \cdot U_{\mathrm{T}}=k_{\mathrm{B}} T / \mathrm{e}$ is the thermal voltage. The existence of shallow acceptors with a density $N_{\mathrm{A}}$ and acceptor-like traps for holes with a density $N_{\text {ta }}$ is supposed. The occupation function of the latter is $f_{\mathrm{t}}$. If occupied, these traps are negatively charged with a concentration $n_{\mathrm{ta}}=N_{\mathrm{ta}} \cdot f_{\mathrm{t}}$. The system of equations is then

$$
\begin{aligned}
\nabla \epsilon \epsilon_{0}(-\nabla \varphi) & =\mathrm{e}\left(p-n-N_{\mathrm{A}}-n_{\mathrm{ta}}\right) \\
\nabla \mathrm{e} p \mu_{\mathrm{p}}\left(-\nabla \varphi_{\mathrm{Fp}}\right) & =-\mathrm{e} \frac{\partial}{\partial t} p-\mathrm{e}\left(U_{\mathrm{SRH}}+U_{\mathrm{pt}}\right) \\
\nabla \mathrm{e} n \mu_{\mathrm{n}}\left(-\nabla \varphi_{\mathrm{Fn}}\right) & =+\mathrm{e} \frac{\partial}{\partial t} n+\mathrm{e} U_{\mathrm{SRH}} \cdot
\end{aligned}
$$

Here $U_{\mathrm{pt}}$ is the net hole capture rate by the traps and $U_{\mathrm{SRH}}$ is the Shockley-Read-Hall recombination rate. Positive voltages larger than the built-in voltage are not considered here, thus direct radiative recombination and other recombination-generation mechanisms are not included. The hole and electron currents are driven by the gradients of the two quasiFermi potentials, but they can be expressed as drift and diffusion currents since the mobilities are connected with the diffusion coefficients $D_{\mathrm{p}}$ and $D_{\mathrm{n}}$ by the (non-degenerate) Einstein relations $\mu_{\mathrm{p}}=D_{\mathrm{p}} / U_{\mathrm{T}}$ and $\mu_{\mathrm{n}}=D_{\mathrm{n}} / U_{\mathrm{T}}$, respectively. These Eq.s (2) do not depend on the type of the transport mechanism and they are especially also valid in the case of hopping transport $^{1}$. It is only assumed that the transport is linear in the gradient of the quasi-Fermi potential. In principle, possible dependencies of the mobilities on concentration and field can be taken into account, but they are not of importance for the processes considered here. Thus, it is just the simple Eq. (1) which requires some care as to be discussed below in subsection III.B.

\footnotetext{
${ }^{1}$ Only in the case of variable range hopping there is the peculiarity that for increasing band bending the type of the hopping mechanism can be changed.
} 
In addition, for the transient behavior the trap rate equation is needed. For an acceptorlike trap with an energy $\varepsilon_{\mathrm{t}}$ several $k_{\mathrm{B}} T$ below the intrinsic energy level $\varepsilon_{\mathrm{i}}$ one has

$$
\begin{aligned}
-\frac{d}{d t} f_{\mathrm{t}} & =\frac{U_{\mathrm{pt}}}{N_{\mathrm{ta}}}=K_{\mathrm{p}} f_{\mathrm{t}}-G_{\mathrm{p}}\left(1-f_{\mathrm{t}}\right) \\
K_{\mathrm{p}} & =\sigma_{\mathrm{p}} v_{\mathrm{th}} p \quad, G_{\mathrm{p}}=\sigma_{\mathrm{p}} v_{\mathrm{th}} n_{\mathrm{i}} \exp \left\{\left(\varepsilon_{\mathrm{i}}-\varepsilon_{\mathrm{t}}\right) / k_{\mathrm{B}} T\right\} .
\end{aligned}
$$

Here $\sigma_{\mathrm{p}}$ is the capture cross section and $v_{\mathrm{th}}$ is the thermal velocity of the holes. $K_{\mathrm{p}}$ is the rate of an occupied trap to capture one hole and $G_{\mathrm{p}}$ is the hole emission rate of an empty trap. This equation is general and applies also to organic semiconductors.

Apart from initial conditions, boundary conditions have to be used at the anode and at the cathode. In the simulations either thermal equilibrium alone (diffusion approximation) or thermal equilibrium in addition with thermionic emission were used and also the image charge correction was been added. It turns out that for the typical parameters described in the following both thermionic emission and the image charge correction lead only to almost negligible modifications. Also, Fowler-Nordheim tunneling observed in different experiments on OLEDs is not of importance in the reverse and low forward bias regime we are interested in here. As barriers at the contacts we choose the differences between the metal work functions and the energy of the transport states $\left(-\chi\right.$ and $-\left(\chi+\varepsilon_{g}\right)$ where $\chi$ is the electron affinity). The total built-in potential is then just the difference between the two metal work functions. Possible dipole layers or Fermi level pinning can be modeled by choosing a corrected work function.

Transient calculations have been carried out for the reverse-to-forward sweep ('up') and for the forward-to-reverse sweep ('down') starting in each case from the corresponding steady-state solutions. Similar as in the experiments, the bias is changed linearly in time during a short ramp time of $2.5 \times 10^{-7} \mathrm{~s}$ by a total amount of the voltage step $\pm \Delta U=0.05 \mathrm{~V}$ (corresponding to a ramp rate of $0.2 \mathrm{~V} / \mu \mathrm{s}$ ). The next ramp follows after the delay time $t_{\mathrm{d}}=2 \mathrm{~s}$. Delay time and voltage step have been varied. 


\section{B. Simulation-relevant material properties}

We emphasize that the phenomenon discussed in this paper can be observed in various types of OLEDs and is therefore not connected with the particular device structure (ITO/NPB/Ca) studied experimentally in detail here. E.g. in devices based on poly(phenylenevinylene) (PPV) we have qualitatively observed the same features [25] without further systematic studies like in the NPB devices described above. Being interested in clarifying the mechanism leading to the hysteresis it is reasonable to choose typical parameters, that means at first a gap of more than $2 \mathrm{eV}$ (as in normal wide-gap semiconductors). However, for a gap larger than $3 \mathrm{eV}\left(\varepsilon_{g}=3.1 \mathrm{eV}\right.$ for NPB [33]) it is well known from simulations for inorganic semiconductors that numerical convergence problems do occur ${ }^{2}$. Thus, we have chosen somewhat different material parameters than expected for NPB, which may be more suitable to an ITO/PPV $/ \mathrm{Al}$ device, i.e. we use an gap energy of $\varepsilon_{g}=2.4 \mathrm{eV}$ as the standard value.

The structure is a 430nm thick organic layer with electrodes on both sides. The width in the $2 \mathrm{D}$ simulation is $1 \mu \mathrm{m}$. Apart from the relative dielectric constant and the electron affinity (typically $\epsilon=3, \chi=2.5 \mathrm{eV}[33]$ ), a further relevant property of organic materials is their low carrier mobility in the range $10^{-7} \ldots .10^{-3} \mathrm{~cm}^{2} / \mathrm{Vs}$ indicating hopping as transport mechanism [34]. As standard parameters we use for the mobilities $\mu_{\mathrm{p}}=10^{-3} \mathrm{~cm}^{2} / \mathrm{Vs}, \mu_{\mathrm{n}}=10^{-5} \mathrm{~cm}^{2} / \mathrm{Vs}$. A possible field dependence $[4,11,13,36]$ of the mobilities for higher fields is not taken into account since the region of interest here is around zero current. There is practically no information on the possible dependence of the mobilities on the carrier concentration for a given doping, which in principle depends on the type of the hopping transport. But dopant and trap concentrations considered here are sufficiently small to neglect such influences. It should be mentioned that the mobilities as used in Eq.s (2) are defined only as averages, e.g. for the holes by the ratio of conductivity $\varkappa_{\mathrm{p}}$ and density, $\mu_{\mathrm{p}}=\varkappa_{\mathrm{p}} / \mathrm{e} p$. The exact values can

\footnotetext{
${ }^{2}$ The reason is the low intrinsic density of less than $100 \mathrm{~cm}^{-3}$ for the gap of NPB.
} 
be regarded as to be determined from fitting e.g. I-V characteristics, however, this mobility is not directly accessible from experiments such as time-of-flight measurements.

The only quantities in the simulation which have to be considered in more detail for organic materials are the concentrations as defined by Eq.s (1). It is supposed that one can divide the whole energy spectrum into current carrying states and trap states. Concerning the former, some different situations will be considered. For a molecular material Eq.s (1) are just the Nernst equations describing oxidation and reduction for low concentrations. As the gap one has the difference between reduction and oxidation potentials (in the solid state). They contain contributions from the deformation of the molecules and possibly entropy contributions for the case of multiple redox sites. The situation is similar in polymers with polarons (and bipolarons) as charged states of the chains, where the gap to be used in Eq. (1) is twice the polaron formation energy [37,38], containing the same influences as just mentioned. In both cases, instead of 'effective densities of states' one has to use the molecular or monomer density which is of the order of $N_{\mathrm{C}}=N_{\mathrm{V}}=5 \times 10^{20} \mathrm{~cm}^{-3}$. The gap must be considered as a phenomenological quantity which is not necessarily equal to the optical gap. In addition, a distribution of the redox potentials or the polaron formation energies characterized by a density of states $\mathcal{N}(\varepsilon)$ can occur. Then one has e.g. for electrons the concentration

$$
\begin{aligned}
n & =N_{\mathrm{C}} \exp \left\{\left(\varepsilon_{\mathrm{F}}-\varepsilon_{\mathrm{c}}\right) / k_{\mathrm{B}} T\right\} \\
N_{\mathrm{C}} & =\int d \varepsilon \mathcal{N}(\varepsilon) \exp \left\{-\left(\varepsilon-\varepsilon_{\mathrm{c}}\right) / k_{\mathrm{B}} T\right\}
\end{aligned}
$$

where $\varepsilon_{\mathrm{c}}$ is the position of the maximum of the distribution. For a width of the distribution small compared to $k_{\mathrm{B}} T$ one obtains for $N_{\mathrm{C}}$ and $N_{\mathrm{V}}$ again the above mentioned values, and smaller ones for broader distributions. If the electrical potential is added Eq. (4) directly leads to Eq.s (1), i.e. the same formal dependence as in a conventional inorganic semiconductor. Two peculiarities have to be mentioned. Although bulk transport is described correctly in this way, the injection into such a distribution results in special effects [19]. But this becomes important only for strong forward bias not considered here. Furthermore, the only 
exception from the expression (4) is an exponential distribution, discussed by several authors [9,18]. But, it can be shown (similar to the derivation in appendix A) for an exponential distribution with a decay energy $\varepsilon_{0}$ much larger than $k_{\mathrm{B}} T$ that the exponential increase of the diode current would be proportional to $\exp \left\{\mathrm{e} U / \varepsilon_{0}\right\}$, whereas in most experiments $j \propto \exp \left\{\mathrm{e} U / n k_{\mathrm{B}} T\right\}$ has been observed with a non-ideality factor around $n \cong 2$. Thus, one can hardly accept such a broad exponential distribution of the transport states.

In organic materials unintentional doping usually leads to either p- or n-type material. Here p-doping by shallow fully ionized acceptors is assumed with a concentration $N_{\mathrm{A}}=$ $10^{14} \mathrm{~cm}^{-3} \ldots 10^{16} \mathrm{~cm}^{-3}$. Concerning these numbers it should be mentioned that even in high quality organic single crystals acceptor concentrations of $10^{13} \mathrm{~cm}^{-3}$ and trap concentrations of $10^{15}$ to $10^{16} \mathrm{~cm}^{-3}$ have been found [35]. As a standard parameter in the simulations $N_{\mathrm{A}}=10^{14} \mathrm{~cm}^{-3}$ is used. Traps at different energies and rather high concentrations have been found in OLEDs [20-23]. Therefore estimates and test calculations have been used to identify the kind of traps and the energy range where traps are important for slow transient processes. Thus, in the simulations acceptor-like traps are taken into account with a concentration $N_{\text {ta }}=4 \times 10^{16} \mathrm{~cm}^{-3}$ and an energy level $\varepsilon_{\mathrm{t}}-\varepsilon_{\mathrm{V}}=0.7 \mathrm{eV}$. Also a Gaussian trap distribution has been considered. If the doping is much smaller than the trap concentration the bulk hole density arises essentially from partially occupied traps. For the trap kinetics according to Eq. (3) we have chosen $\sigma_{\mathrm{p}} v_{\mathrm{th}}=10^{-14} \mathrm{~cm}^{3} / \mathrm{s}$ corresponding to a hole capture cross section $\sigma_{\mathrm{p}}=10^{-15} \mathrm{~cm}^{2}$ and a rather small thermal velocity $v_{\text {th }}=10 \mathrm{~cm} / \mathrm{s}$ for the hopping conduction with the above mentioned mobility [6,39]. Fast shallow trap states are not included as they are important only for larger positive bias in the space-charge limited region we are not considering here.

As the cathode work function $\Phi_{\mathrm{C}}=4.1 \mathrm{eV}(\mathrm{Al})$ has been chosen. The anode is simulated usually as a neutral contact. Larger anode work functions have also been chosen, they lead to a hole accumulation layer resulting in space-charge limited transport for a bias above the built-in voltage, but the modification is rather unimportant for reverse and small forward bias considered here. 
To establish systematic trends most of the material parameters have been varied in a reasonable range. The simulations have shown that for reverse and small forward bias and for the typical material parameters the SRH recombination $\left(\tau_{\mathrm{n}}=\tau_{\mathrm{p}}=10^{-7} \mathrm{~s}\right)$ is negligible. The same holds for thermionic injection (hence the surface recombination velocity) and inclusion of the image charge correction.

\section{SIMULATED INFLUENCE OF DEEP TRAPS}

Simulated currents are given in the following as current density $j$ in units of $\mathrm{A} / \mathrm{cm}^{2}$. In the graphical representations of field and concentration distributions the anode is at $x=0$ and the cathode at $x=430 \mathrm{~nm}$. Instead of electrical potentials (in units of $\mathrm{V}$ ) used in Eq.s (1), (2) the corresponding energies (position dependent intrinsic level and quasi-Fermi energy) are depicted below.

The principal effect of slow recharging of traps on the transient I-V characteristics will be discussed in detail for the 'up' sweep illustrated by the results shown in Fig.s 4 and 5. The parameters of the doping concentration and the traps are chosen as described in the previous section. An anode work function of $4.7 \mathrm{eV}$ is assumed here leading to a hole accumulation layer. The transient simulation started from the steady-state solution at $U=$ $-1.5 \mathrm{~V}$ with the delay time $t_{d}=2 \mathrm{~s}$. Fig. $4 \mathrm{a}$ shows the simulated I-V characteristic (i.e. the current after ramp plus delay time as in the measurements). It is clearly seen that the voltage position of the current zero is shifted now from the steady-state value at $U=0 \mathrm{~V}$ to $U=-1.05 \mathrm{~V}$. Since the simulation starts at $U=-1.5 \mathrm{~V}$ the current at the end of the delay time becomes zero at $t=18 \mathrm{~s}$ as seen in Fig. 4c. Fig.s $4 \mathrm{~b}$ and $4 \mathrm{c}$ show the current as a function of time on different scales. One can see here, how the current changes during the ramp and delay time, respectively. During the ramp time (Fig. 4b) there is always the same positive displacement current (narrow peak) of about $1.3 \mathrm{~mA} / \mathrm{cm}^{2}$ yielding an areal capacity of $C=j / v_{\mathrm{V}} \approx 6.5 \mathrm{nF} / \mathrm{cm}^{2}$ where $v_{\mathrm{V}}$ is the ramp rate. For comparison, the geometric capacity is $6.2 \mathrm{nF} / \mathrm{cm}^{2}$ (the effective layer thickness is somewhat smaller than 
the geometric value because of the accumulation layer at the anode side). After the ramp time the current shows a short overshoot to negative values (here about $-0.3 \mathrm{~mA} / \mathrm{cm}^{2}$ ) and should then become constant and equal to the steady-state current in the absence of traps. With traps, however, as demonstrated in Fig. 4c, the current is at first negative and almost constant. With increasing voltage it becomes positive at the beginning of the delay time and decreases slowly towards the steady-state value. However, since the measurement occurs already after $2 \mathrm{~s}$ (before the next ramp occurs) the measured current is zero already after 18 s corresponding to a negative bias of $U=-1.05 \mathrm{~V}$. From this picture it is intuitively clear that increasing the delay time will give the current more time to approach the steady-state value and thus reduce the shift of the measured zero crossing towards $U=0 \mathrm{~V}$.

The internal potential, concentration and current density profiles of the device of Fig. 4 are depicted in Fig. 5 for one applied voltage $(U=-0.5 \mathrm{~V})$ after the delay time. Position dependent energies (valence and conduction band edges, the trap energy level and the hole quasi-Fermi level) are drawn in Fig. 5a, the hole concentration and the concentration of the trap occupancy ${ }^{3}$ in Fig. 5b and the hole current density in Fig. 5c, respectively. Near the anode $(x=0)$ at the left-hand side there is a hole accumulation and on the right-hand side near to the cathode $(x=0.43 \mu \mathrm{m})$ a depletion layer connected with fully occupied traps there. In this region the hole quasi-Fermi level lies above the trap energy level. At the position where the trap energy and the hole quasi-Fermi levels are crossing each other half of the traps are occupied. At this intercept point the recharging of the traps is expected to be most important. It should be mentioned here that, although the simulation program actually solves the complete system of equations, there is up to now only the possibility to extract from the program as trap occupancy the equilibrium value corresponding to the actual transient hole concentration. This is shown in Fig. 5b. The time evolution of the

\footnotetext{
${ }^{3} n_{\mathrm{ta}}=N_{\mathrm{ta}} f_{\mathrm{t}}$ is the concentration of acceptor-like traps which are occupied with electrons and can therefore capture holes, see Eq. (3)).
} 
trap occupancy cannot be demonstrated here. More information is obtained from the hole current (Fig. 5c). For negative bias one has in the steady-state a negative and position independent hole current in the whole device. Increasing the bias in the up sweep, the extension of the depletion layer must be reduced. Consequently this must be connected with a hole capture by the traps. This process is slow and is therefore not finished after the delay time considered here. But the actual hole current after the delay time flows from both the anode and the cathode to those traps which are capturing them (just at $p=n_{\mathrm{ta}}$ ). Thereby the integral hole current is dominated by the positive component as if the device would already have been forward biased in spite of the actual value $U=-0.5 \mathrm{~V}$ (see. also Fig. 4).

The effect caused by the recharging of the traps leads to the shift of the current zero only when the recharging current at the end of the delay time is comparable to the steady-state reverse current. Using Eq. (3) one can try to estimate the recharging relaxation time $\tau$. Immediately after the voltage ramp, capture dominates which is described by the first term on the right-hand side of Eq. (3). If after a (sufficiently short) delay time recharging is by far not finished, this term can be used to estimate the relaxation time using simulated concentrations. Evidently, due to the dependence on the hole density there is no constant relaxation time through the device. For a simple estimation one can consider at first the position where the quasi-Fermi and the trap energy levels are crossing each other and where one could expect recharging do be most important. In this case one has a hole density $p_{1} \equiv p\left(\varepsilon_{\mathrm{ta}}=\varepsilon_{\mathrm{F}}\right) \approx 10^{9} \mathrm{~cm}^{-3}$ leading to $\tau=\left(\sigma_{\mathrm{p}} v_{\mathrm{th}} p_{1}\right)^{-1} \approx 10^{5} \mathrm{~s}$. Actually, holes flow from both sides to the position where the hole current vanishes which seems to be more likely the position of most effective recharging. There the hole density is $10^{13} \mathrm{~cm}^{-3}$ (see Fig.5). In contrast to the former case, the relaxation time is then $\tau \approx\left(\sigma_{\mathrm{p}} v_{\mathrm{th}} p\right)^{-1} \approx 10 \mathrm{~s}$. Thus one cannot introduce a general time constant for trap recharging. Furthermore, the actual time dependence of the recharging process as described by Eq. (3) depends on the trap occupation associated with both capture and emission processes. Nevertheless, these estimates show that $\tau$ is of the order of tens of seconds. 
The trap relaxation time alone is not sufficient to understand the shift of the current zero. In order to demonstrate this effect, in Fig. 6 the thermal velocity is chosen as in Si $\left(v_{\text {th }}=10^{7} \mathrm{~cm} / \mathrm{s}\right)$, i.e. six orders of magnitude larger than in organic materials. For the anode a neutral contact is used apart from one curve in Fig. 6a (open circles) where the same anode contact as in Fig. 4 has been chosen for comparison. Although $v_{\text {th }}$ enters linearly into the kinetic equation (3) the shift of the current zero is reduced only from $-1.05 \mathrm{~V}$ (Fig. 4) to $-0.7 \mathrm{~V}$ (Fig. 6a). This is due to the fact that the actual reason for the shift of the current zero is not only the slowly decreasing current for recharging of the traps but also the different voltage dependencies of the steady-state negative current and of the positive trap recharging current at the end of the delay time. It is hardly possible to find a simple estimate for the latter. The steady-state reverse current is proportional to the carrier mobility. Hence the shift of the current zero will disappear or will be smaller at least for a larger mobility. Furthermore, Fig. 6 shows the influence of various parameters on the I-V characteristics. The role of the anode work function is shown in Fig. 6a in comparison with the steadystate current. For the anode work function of $4.7 \mathrm{eV}$ there is an accumulation layer near to the anode. This layer influences the shift of the current zero only slightly, but in forward direction it leads to space-charge limitation of the current (otherwise, in this region one has to consider additional effects which is not done here). In Fig. $6 \mathrm{~b}$ the trap energy level is varied from $0.5 \mathrm{eV}$ to $0.9 \mathrm{eV}$. For the parameter set of this device the trap energy level of $0.7 \mathrm{eV}$ is the value leading to the largest shift of the current zero to $U=-0.8 \mathrm{~V}$. Lower trap energies lead to faster recharging processes, whereas for too high trap energies there is no recharging at all since the quasi-Fermi level is not crossing the trap level. In both cases the shift of the current zero is reduced. In the situation depicted in Fig. 6b the trap energy of $0.5 \mathrm{eV}$ is already too low, the recharging is so fast that the shift of the current zero disappears. Whether the trap energy level is too high or too low depends on the cathode work function determining the band bending and hence the crossing of the trap level with the quasi-Fermi level. For the trap energy level of $0.9 \mathrm{eV}$ the recharging is essentially less than for $0.7 \mathrm{eV}$. The shift of the current zero moves to $U=-0.4 \mathrm{~V}$. The influence of the 
doping concentration is demonstrated in Fig. 6c. Larger doping of the order of the trap concentration reduces the influence of the traps and hence the current zero shift becomes smaller. With $N_{A}=10^{12} \mathrm{~cm}^{-3}$ the shift of the current zero is at $U=-0.9 \mathrm{~V}$ and at the same time the device has a low rectification. The value of the current at $U=2 \mathrm{~V}$ is only four orders of magnitude larger than at $U=-1.5 \mathrm{~V}$. With $N_{A}=10^{14} \mathrm{~cm}^{-3}$ the current zero is shifted slightly to the right hand side at $U=-0.8 \mathrm{~V}$. However the value of the current in the forward direction is already two orders of magnitude larger. This tendency is seen more pronounced for $N_{A}=10^{16} \mathrm{~cm}^{-3}$. In contrast, the influence of the recharging current increases with the trap concentration as demonstrated in Fig. 6d. When the trap concentration is one order of magnitude smaller $\left(N_{t a}=4 \times 10^{15} \mathrm{~cm}^{-3}\right)$ the shift of the current zero moves from $U=-0.8 \mathrm{~V}$ to $U=-0.4 \mathrm{~V}$. For one order of magnitude larger trap concentration $\left(N_{t a}=4 \times 10^{17} \mathrm{~cm}^{-3}\right)$ the current passes through zero already at $U=-1.05 \mathrm{~V}$. It is seen that deep traps do not influence the value of the current in forward direction. The value of the current for $U>0.5 \mathrm{~V}$ is the same for all three trap concentrations.

The internal field and concentration distributions at the end of the delay time, corresponding to Fig. 6d, are shown in Fig. 7 at $U=-0.5 \mathrm{~V}$. The crossing of the hole quasi-Fermi level with the trap level (Fig. 7a) marks the extension of the depletion layer since the basic acceptor doping is much lower than the trap concentration, and the width of this layer increases with decreasing trap concentration. The trap occupancy shown in Fig. 7c (as mentioned above, this is the equilibrium distribution corresponding to the transient hole concentration shown in fig. $7 \mathrm{~b}$ ) reaches the nominal trap concentration just in the depletion region. Although these internal distributions determine in detail the processes leading to the shift of the current zero one cannot draw direct conclusions on this shift from them.

A Gaussian distribution of traps has been discussed by several authors [19,23]. In Fig. 8 we demonstrate the influence of such a trap distribution on the I-V characteristics in comparison with a discrete trap level. The parameters $N_{A}=10^{16} \mathrm{~cm}^{-3}, N_{t a}=4 \times 10^{16} \mathrm{~cm}^{-3}$, $v_{t h}=10 \mathrm{~cm} / \mathrm{s}$ and a neutral anode have been chosen. For the discrete trap energy level $\varepsilon_{\mathrm{ta}}-\varepsilon_{\mathrm{V}}=0.7 \mathrm{eV}$ is used where approximately the largest shift of the current zero does 
occur as shown above. The Gaussian distribution of the traps around this value has a width of $0.1 \mathrm{eV}$. These parameters approximately correspond to the situation in PPV [23]. For the Gaussian distribution the traps with higher energy $(>0.7 \mathrm{eV})$ will be less effectively recharged, but their recharging process during the delay time is slower. In total, the shift of the current zero is only a little smaller than for the discrete level.

A comparison of the I-V characteristics for different delay times varying from $1 \mathrm{~s}$ to 10s is presented in Fig. 9. Here also the up and down sweeps are compared with each other. The parameters of the device are the standard values as described in the preceding section but the trap energy level $\varepsilon_{\mathrm{ta}}-\varepsilon_{\mathrm{V}}=0.5 \mathrm{eV}$ is chosen. The experimentally observed trend is clearly obtained here. In the up sweep (Fig. 9a) the shift of the current zero is reduced with increasing delay time, and above some positive voltage (here $U \approx 0.5 \mathrm{~V}$ ) all the characteristics approach the steady-state values. In the down sweep (Fig.9b) the negatively biased current is proportional to the applied voltage and its value decreases with increasing delay time as in the experiment. All the I-V characteristics in the down sweep do exhibit the steep increase around zero bias and not at a positive bias as in the experiment. The mechanism in the down sweep is in principle the same as in the up sweep, but instead of the slow hole capture there is now hole emission in order to extend the space-charge layer with decreasing bias. The recharging current is then negative, hence the current zero must occur indeed at a positive bias. However, in practice the steady-state current increases almost exponentially with the applied bias already from $U=0 \mathrm{~V}$ and becomes much larger than the recharging current. Hence one cannot see the shift of the current zero here.

As already mentioned, for numerical reasons the simulations have been carried out for a material with a band gap of $2.4 \mathrm{eV}$ and $\mathrm{Al}$ as cathode. The simulated rectification ratio is in all cases of the order $10^{6}$ similar to the experiments shown in Fig.s 1 and 2. However, the actual band gap of NPB is approximately $3.1 \mathrm{eV}$ and in addition the low-work function Ca has been used as the cathode. With these values the steady-state reverse diode current should be many orders of magnitude smaller as demonstrated in the appendix for appropriate values of the barrier and the built-in potential. Therefore the comparatively high reverse-bias currents 
in the experiment can only be interpreted as leakage currents. As shown in the appendix such a leakage current, which may be approximately Ohmic, naturally leads to the reduced rectification ratio and at the same time to the steep increase in the $\mathrm{I}-\mathrm{V}$ characteristics not at 0V but at a finite positive voltage. In the appendix an analytical approximation (Eq. (A3)) for the diode current density $j$ in the case of a fully depleted layer is derived. Using this expression the total current can be expressed as $j_{\text {tot }}=j+j_{\text {leakage }} \pm j_{\text {trap }}$, where in the simplest approximation the leakage current depends linearly on the voltage and the trap recharging current is bias independent (the positive/negative sign corresponds to the up/down sweep). This approximation has been used to describe the experimental results for both the up and down sweep shown as solid lines in Fig. 3 with $U_{\mathrm{bi}}=2.75 \mathrm{~V}$ which is larger than the work function difference between cathode and anode. We can only speculate that this difference might be caused by some kind of additional dipole barrier. In addition, in the last nominator and denominator of Eq. (A3) the ideality factor $n=2.5$ has been introduced by replacing $k T$ with $n k T$. The fitted conductance of the leakage current is $1.5 \times 10^{-12} \mathrm{~A} / \mathrm{V}$ which is much larger than the reverse current expected for a diode with a large hole barrier at the cathode (see Appendix). This leakage current is also seen in the $\mathrm{I}-\mathrm{V}$ characteristics above $U=0 \mathrm{~V}$ up to the turn-on voltage. The trap recharging current density is $8.75 \times 10^{-12}$, $1.09 \times 10^{-11}, 3.75 \times 10^{-10}, 1 \times 10^{-9} \mathrm{~A} / \mathrm{cm}^{2}$ for decreasing delay times as indicated in Fig. 3. For the largest delay time this is small compared to the leakage current apart from bias values close to $0 \mathrm{~V}$ and the shift of the current zero is small. The agreement of this simple approximation with the experimental curves is rather good for larger delay times in the up sweep and for the down sweep for all delay times except from a small bias region where the transition from depletion to the flat band case occurs and hence the assumption of a bias independent trap recharging current is not sufficient. The same is true for shorter delay times in the up sweep. The prefactor in Eq. (A3), $A \mathrm{e} N_{\mathrm{v}} \mu d^{-1} \exp \left(-\frac{V_{\mathrm{B}}}{k T}\right)$, with the device area $A$ is $10^{-24} \mathrm{~A} / \mathrm{V}$. A reasonable estimate of the barrier from this expression is difficult essentially due to the phenomenological introduction of the ideality factor. Nevertheless, the approximation introduced here can be useful in analyzing experimental data. 


\section{CONCLUSIONS}

It has been shown by simulations that the experimentally observed hysteresis in the I$\mathrm{V}$ characteristics of OLEDs with a shift of the current zero in opposite direction for the up and down sweep is caused by the slow recharging of deep traps, which in the case of a p-material must be acceptor-like. Several conditions must be fulfilled for the occurrence of the shift. At first, only low mobilities as they occur for hopping conduction in organic materials lead to a sufficiently small reverse current that the trap recharging current becomes comparable or even larger. Further, it is important for the occurrence of the effect that the trap recharging current (at the end of the delay time between the application of the voltage and the measurement of the current) and the steady-state reverse current have a different bias dependence. Next, there must be a sufficiently high concentration of traps (arising e.g. from disorder or being caused by chemical impurities, especially due to oxygen exposure). This concentration should be comparable with or even larger than the doping with relatively flat acceptors. Indeed, the materials used for OLEDs are usually only unintentionally doped (apart from few attempts to modify hole transport layers by doping [40]). The extremely slow recharging requires very deep traps which are possible only in wide-gap materials (which is not exclusively typical for organics, e.g. $\mathrm{SiC}$ and $\mathrm{GaN}$ have similar gaps but they have much higher mobilities). Since the trap recharging requires a crossing between the trap level and the quasi-Fermi level not only the trap energy itself but also the band bending at the cathode is essential. Thus, depending on the cathode contact, on the one hand for a slow recharging deep traps are needed, but on the other hand, if they lie too deep, there is no recharging at all. According to the simulations a distribution of the trap energy of moderate width (around 100meV) modifies the shift of the current zero only slightly. Considering all these influences and the fact that the shift of the current zero has been observed for rather different materials, cathode contacts and mobilities, one can conclude that there is

usually a rather broad distribution of the trap energies such that a considerable part of them contributes to the slow processes. The large time constant is also favored by the 
small thermal velocity in the case of hopping transport. Due to the combined effects of different influences, especially the required compensation of reverse steady-state and trap recharging currents, it is hardly possible to extract trap or material parameters immediately from the measured dependencies. In addition, the almost Ohmic behaviour of the current for small positive bias before the current begins to increase exponentially could not be simulated without the additional assumption of leakage currents. Indeed, due to the large hole barrier at a cathode with low work function and, at the same time, the small mobility of the organic semiconductor, the true steady-state reverse bias diode current should be many orders of magnitude smaller than the measured ones. This can be explained only by additional quasi-Ohmic leakage currents. With this assumption the steep increase of the I-V characteristics is shifted from $0 \mathrm{~V}$ to a finite positive bias and the current zero occurs at a positive voltage in the down sweep. All these dependencies are described rather well by using the approximation for the current of a completely depleted diode derived in the Appendix and phenomenologically adding an Ohmic leakage current and a trap recharging current. The latter is for not too large delay times and in the region of trap depletion approximately independent of the bias. The condition of complete depletion is usually fulfilled in OLEDs since for only unintentional doping and low trap concentrations the depletion length is

much larger than the typical layer thickness of the order of $100 \mathrm{~nm}$. Finally, the details of the simulations and of the phenomenological modeling in comparison with the experiment show that the shift of the current zero can be explained by deep traps and leakage currents without any contribution of mobile ions, nevertheless their existence is not excluded in this way.

\section{ACKNOWLEDGMENTS}

The authors thank Professor Markus Schwoerer for his continuous interest in the work and the Deutsche Forschungsgemeinschaft for financial support. 


\section{APPENDIX: THE DEPLETED SCHOTTKY DIODE AND THE INFLUENCE OF A LEAKAGE CURRENT}

The common expression from diffusion theory for the Schottky diode current for a bias below the built-in voltage $\left(U<V_{\mathrm{bi}} / \mathrm{e}\right)$ is for a p-material [41]

$$
j=\mathrm{e} \mu N_{\mathrm{v}} \exp \left(-\frac{V_{\mathrm{B}}}{k T}\right) \sqrt{\frac{2 N_{A}^{-} \mathrm{e}}{\varepsilon \varepsilon_{0}}\left(U_{\mathrm{bi}}-U\right)}\left(\exp \left(\frac{\mathrm{e} U}{k T}\right)-1\right) .
$$

Here $N_{A}^{-}$is the concentration of the ionized acceptors, the hole barrier $V_{\mathrm{B}}$ is the difference between the cathode work function and the valence band edge (eventually corrected for the occurrence of surface dipoles or due to Fermi level pinning) and the built-in energy $V_{\mathrm{bi}}=\mathrm{e} U_{\mathrm{bi}}$, the difference between the cathode work function and the semiconductor Fermi energy. The main assumptions leading to (A1) are: non-degeneration, constant mobility, cathode in thermal equilibrium (no thermionic emission i.e. a high barrier), electron current and recombination negligible and, usually not mentioned explicitly, a thickness of the diode large compared to the width of the depletion layer. As demonstrated in Fig. A1 the simulated current (thermionic emission is indeed negligible as proved by the simulation) for a sufficiently high doped OLED is described well by Eq. (A1) only for negative bias, whereas one has already a strong deviation for $0<U<V_{\mathrm{bi}} /$ e. However, since a typical OLED, with only unintentional doping and less then 100nm thickness is completely depleted,

the potential varies almost linearly between cathode and anode and the built-in energy is now the difference between cathode and anode work function (for a supposed neutral anode contact). With the remaining assumptions as before and expressing the current by the gradient of the quasi-Fermi level, separation of variables leads to

$$
j \times \int_{0}^{d} \exp \left(\frac{V_{\mathrm{v}}(x)}{k T}\right) d x=\operatorname{e} \mu N_{\mathrm{v}} k T\left(\exp \left(\frac{U}{k T}\right)-1\right)
$$

and, considering the approximately linear position dependence of the valence band edge $V_{\mathrm{v}}(x)$, one obtains instead of Eq. (A1) now

$$
j=\mathrm{e} N_{\mathrm{v}} \mu \frac{U_{\mathrm{bi}}-U}{d} \exp \left(-\frac{V_{\mathrm{B}}}{k T}\right) \frac{\exp \left(\frac{\mathrm{e} U}{k T}\right)-1}{1-\exp \left(\mathrm{e} \frac{U-U_{\mathrm{bi}}}{k T}\right)} .
$$


In contrast to Eq. (A1), this expression is valid below and above the built-in voltage. Below zero and above the built-in potential the current in Eq. (A3) becomes Ohmic according to

$$
\begin{aligned}
& j=-\mathrm{e} N_{\mathrm{v}} \exp \left(-\frac{V_{\mathrm{B}}}{k T}\right) \mu \frac{U_{\mathrm{bi}}-U}{d}, \quad-U \ll k T / \mathrm{e} \\
& j=+\mathrm{e} N_{\mathrm{v}} \exp \left(-\frac{V_{\mathrm{B}}-V_{\mathrm{bi}}}{k T}\right) \mu \frac{U-U_{\mathrm{bi}}}{d} \quad, \quad U-U_{\mathrm{bi}} \gg k T / \mathrm{e}
\end{aligned}
$$

It is shown in Fig. A1 that the approximation (A3) yields good agreement of the simulated current for a low doped and hence almost depleted diode. In Eq. (A3) the input parameters are the same as used in the simulation, especially the barrier is $0.8 \mathrm{eV}$ and the built-in potential for the given dopant and trap density and for the assumed neutral anode contact is $0.402 \mathrm{eV}$. In practice, one usually does not have a neutral anode but hole injection due to a thin hole accumulation layer (sufficiently large anode work function). In this case Eq. (A3) has to be restricted to $U<U_{\mathrm{bi}}$ and for $U>U_{\mathrm{bi}}$ the usual approximation for space-charge limited transport can be used.

In OLEDs with a low work function cathode (some tenths of an $\mathrm{eV}$ less than for $\mathrm{Al}$ ) the current for reverse bias is for both Eqs. (A1) and (A4) many orders of magnitude less than the measured one and even much less than the experimental measuring limit. Moreover, the measured current is approximately Ohmic in reverse bias direction and for a positive voltage up to about $2 \mathrm{eV}$ (see Fig.1) before the exponential increase begins, whereas in a normal diode this exponential increase begins already a few $k T /$ e above zero. It is supposed that such a behaviour is caused by an additional leakage current which has already been discussed for GaAs Schottky diodes [42]. Assuming that the total current density is composed of the diode current ( $j$ from Eq. A3)) and an Ohmic leakage current (with a conductance of $10^{-13}$ in the reduced units of the figure), it is demonstrated in Fig. A2 (with $V_{\mathrm{B}}=1.9 \mathrm{eV}$ and $V_{\mathrm{bi}}=1.5 \mathrm{eV}$ ) that the total current density $j_{\mathrm{tot}}=j+j_{\text {leakage }}$ follows Ohmic behaviour for $U-U_{\mathrm{bi}} \gg k T / \mathrm{e}(\mathrm{A} 5)$ and that the exponential decrease of $j$ for decreasing bias below the built-in voltage intersects at about $1.2 \mathrm{~V}$ with the additional Ohmic leakage current. 


\section{REFERENCES}

[1] C.W. Tang and S.A. Van Slyke, Appl. Phys. Lett. 51 (1987) 913.

[2] C J. Burroughes, D.D.C. Bradley, A.R. Brown, R.N. Marks, K. Mackey, R.H. Friend, P.L.Burn, and A.B. Holmes, Nature 347 (1990) 539.

[3] Pioneer Co. (Japan). In November 1997 Pioneer Co. in Japan commercialized a monochrome $256 \times 64$ dot matrix OLED display for automotive applications.

[4] P.W.M. Blom, M.J.M. de Jong, and C.T.H.F. Liedenbaum, Polymers For Advanced Technologies 9 (1998) 390.

[5] J. Staudigel, M. Stößel, F. Steuber, and J. Simmerer, J. Appl. Phys. 86 (1999) 3895.

[6] A.J. Campbell, D.D.C. Bradley, and D.G. Lidzey, J. Appl. Phys. 82 (1997) 6326.

[7] A.J. Campbell, M.S. Weaver, D.G. Lidzey, and D.D.C. Bradley, J. Appl. Phys. 84 (1998) 6737.

[8] P.E. Burrows, Z. Shen, V. Bulovic, D.M. McCarty, S.R. Forrest, J.A. Cronin, and M.E. Thompson, J. Appl. Phys. 79 (1996) 7991.

[9] J. Shen and J. Yang, J. Appl. Phys. 83 (1998) 7706.

[10] M. Stößel, J. Staudigel, F. Steuber, J. Blässing, J. Simmerer, and A. Winnacker, Appl. Phys. Lett. 76 (2000) 115.

[11] P.W.M. Blom, M.J.M. de Jong, and M.G. van Munster, Phys. Rev B 55 (1997) R656.

[12] P.W.M. Blom and M.C.J.M. Vissenberg, Phys. Rev. Lett. 80 (1998) 3819.

[13] A. Ioannidis, E. Forsythe, Y. Gao, M.W. Wu,and E.M. Conwell, Appl. Phys. Lett. 72 (1998) 3038 .

[14] J.C. Scott, S. Karg, and S.A. Carter, J. Appl. Phys. 82 (1997) 1454.

[15] W. Brütting, S. Berleb, and A.G. Mückl, Synth. Met. 122 (2001) 99. 
[16] P.S. Davids, I.H. Campbell, and D.L. Smith, J. Appl. Phys. 82 (1997) 6319.

[17] M.C.J.M. Vissenberg and M. Matters, Phys. Rev B 57 (1998) 12964.

[18] J. Yang and J. Shen, J. Appl. Phys. 84 (1998) 2105.

[19] V.I. Arkhipov, U. Wolf, and H. Bässler, Phys. Rev B 59 (1999) 7507\&7514.

[20] M. Meier, K. Zuleeg, S. Karg, W. Brütting, and M. Schwoerer, J. Appl. Phys. 84 (1998) 87.

[21] S. Karg, J. Steiger, and H. von Seggern, Synthetic Metals 111-112 (2000) 277.

[22] E. W. Forsythe, D.C. Morton, C.W. Tang, and Y. Gao. Appl. Phys. Lett. 73 (1998) 1457.

[23] A.J. Campbell, D.D.C. Bradley, E. Werner, and W. Brütting, Synth. Met 111-112 (2000) 273.

[24] S. Berleb, W. Brütting, and G. Paasch, Organic Electronics 1 (2000) 41.

[25] W. Brütting, M. Meier, M. Herold, S. Karg and M. Schwoerer, Chem. Phys. 227 (1998) 243.

[26] W. Brütting, H. Riel, T. Beierlein, and W. Rieß, J. Appl. Phys. 89 (2001) 1704.

[27] T. Feng, T.A. Ali, E.S. Ramakrishnan, R. Campos, and W.E. Howard, SPIE Vol. 4105 (2001) 30.

[28] J.C. deMello, N. Tessler, S.C. Graham, and R.H. Friend, Phys. Rev B 57 (1998) 12951.

[29] D. Zou, M. Yahiro, and T. Tsutsui, Appl. Phys. Lett. 72 (1998) 2484.

[30] G. Paasch and S. Scheinert, Synthetic Metals 122 (2001) 145.

[31] S. Scheinert, G. Paasch, P.H. Nguyen, S. Berleb, and W. Brütting, Proc. ESSDERC 2000, Edited by Frontier Group, p. 444-447. 
[32] ATLAS User's Manual: Device Simulation Software, SILVACO International, Santa Clara. Version 1.5.0 (1997).

[33] I.G. Hill and A. Kahn, SPIE Vol. 3476 (1998) 168.

[34] P.M. Borsenberger and D.S. Weiss, Organic Photoreceptors for Imaging Systems, Marcel Dekker, New York 1993.

[35] J.H. Schön, C. Kloc, R.A. Laudise, and B. Batlogg, Phys. Rev. B 58 (1998) 12952.

[36] A.G. Mückl, S. Berleb, W. Brütting, and M. Schwoerer, Synth. Met. 111-112 (2000) 91.

[37] P.S. Davids, A. Saxena and D.L. Smith, J. Appl. Phys. 78 (1995) 4244.

[38] G. Paasch, P.H. Nguyen, and A. Fisher, Chem. Phys. 227 (1998) 219.

[39] S. Scheinert, G. Paasch, and R. Tecklenburg, Proc. ESSDERC'98, Editions Frontiers (1998), p. 628.

[40] M. Pfeiffer, A. Beyer, T. Fritz, and K. Leo, Appl.Phys.Lett. 73 (1998) 3202.

[41] S.M. Sze, Physics of Semiconductor Devices, Second Edition, John Wiley \& Sons, New York 1981.

[42] J.A. Ellis and P.A. Barnes, Appl. Phys. Lett. 76 (2000) 124. 


\section{Captions}

Fig. 1. Schematic drawing of the procedure performed to measure transient I-V characteristics of OLEDs.

Fig. 2. I-V characteristics of an ITO/NPB/Ca single-layer device with a 430nm thick NPB layer measured with voltage steps of $0.1 \mathrm{~V}$ and different delay times. The hold time used after application of the first voltage value $(-7 \mathrm{~V})$ is the same as the delay time. The arrows indicate the sweep direction. The inset shows the current measured with the delay time of 300s for $U<2 \mathrm{~V}$ on a linear scale.

Fig. 3. I-V characteristics of the same device as in Fig. 2 measured with voltage steps of $0.1 \mathrm{~V}$, different delay times for reverse-to-forward (a) and forward-to-reverse bias sweep (b). Here an additional hold time of 655 s was used after application of the first voltage value. The solid lines indicate fits with our analytical model with leakage and trap recharging currents as described at the end of Section IV.

Fig. 4. Simulated I-V characteristics for reverse-to-forward bias sweep with standard parameters: $N_{A}=10^{14} \mathrm{~cm}^{-3}, N_{\mathrm{ta}}=4 \times 10^{16} \mathrm{~cm}^{-3}, \varepsilon_{\mathrm{ta}}-\varepsilon_{\mathrm{V}}=0.7 \mathrm{eV}$ and an anode work function of $4.7 \mathrm{eV}$. The upper graph (a) shows the current density after a delay time of $2 \mathrm{~s}$. The lower two graphs show the time dependence (from $6 \mathrm{~s}$ to $24 \mathrm{~s}$ : $-1.35 \mathrm{~V}$ to $-0.9 \mathrm{~V})$ of $(\mathrm{b})$ the capacitive current during the ramp time and $(\mathrm{c})$ the trap recharging current during the delay time.

Fig. 5. Profiles of energies (valence and conduction band edges, hole quasi Fermi level and trap level) (a), hole concentration and concentration of the trap occupancy (b) and the hole current density (c) of the device in Fig. 4 at the applied voltage of $-0.5 \mathrm{~V}$ after the delay time. The anode is on the left hand side, the cathode right.

Fig. 6. Influence of different material parameters on the I-V characteristics for reverse-toforward sweep direction. Only one of the basic material parameters is varied in each 
picture: (a) anode work function, (b) trap energy level, (c) doping concentration and (d) trap concentration.

Fig. 7. Profiles of the quasi-Fermi and trap energy level (a), of the hole and electron concentrations (b) and of the trap occupancy (c) at the applied voltage of $-0.5 \mathrm{~V}$ after the delay time for various trap concentrations.

Fig. 8. Influence of the trap distribution on the transient and steady-state I-V characteristic. The solid line is the current density calculated with the basic parameters $\left(\epsilon=3, \varepsilon_{g}=2.4 \mathrm{eV}, \chi=2.5 \mathrm{eV}, \mu_{n}=10^{-5} \mathrm{~cm}^{2} / \mathrm{Vs}, \mu_{p}=10^{-3} \mathrm{~cm}^{2} / \mathrm{Vs}\right.$, $N_{C}=N_{V}=5 \times 10^{20} \mathrm{~cm}^{-3}, N_{A}=10^{14} \mathrm{~cm}^{-3}, N_{\mathrm{ta}}=4 \times 10^{16} \mathrm{~cm}^{-3}, \varepsilon_{\mathrm{ta}}-\varepsilon_{\mathrm{V}}=0.7 \mathrm{eV}$ and a neutral anode). For the dashed line the discrete trap level is replaced by a Gaussian distribution with a maximum energy at $0.7 \mathrm{eV}$ above the valance band and a width of $0.1 \mathrm{eV}$. The dotted line indicates the steady-state value.

Fig. 9. Simulated current density in up (a) and down sweep (b) for various delay times. The parameters are chosen as in Fig. 8 , but $\varepsilon_{\mathrm{ta}}-\varepsilon_{\mathrm{V}}=0.5 \mathrm{eV}$.

Fig. A1. Comparison of the simulations (standard parameters as in Fig.8, trap and doping concentrations as indicated) with the standard diffusion model Eq. (A1) and the model for the depleted diode Eq. (A3).

Fig. A2. Current density (in reduced units) of the depleted diode, Eq. (A3), without (dashed) and with (solid) an additional Ohmic leakage current (conductance $10^{-13}$ in reduced units) for typical values of the barrier and the built-in potential. Without leakage the current goes down to $10^{-32}$ at $-1 \mathrm{~V}$ due to the large barrier. Above the built-in potential there is a linear dependence (right scale). 